\title{
Low-noise 16-bit First-order Delta-sigma Capacitance-to-digital Converter for Capacitive Humidity Sensor
}

\author{
Byeoncheol Lee, ${ }^{1}$ Hyungseup Kim, ${ }^{1}$ Jaesung Kim, ${ }^{1}$ Kwonsang Han, ${ }^{1}$ Sangyoun Shin, ${ }^{2}$ \\ Gyungtae Kim, ${ }^{3}$ Woo Suk Sul, ${ }^{3}$ Boung Ju Lee, ${ }^{3}$ Sangmin Lee, ${ }^{4}$ and Hyoungho Ko ${ }^{1 *}$ \\ ${ }^{1}$ Department of Electronics Engineering, Chungnam National University, Daejeon 34134, Republic of Korea \\ ${ }^{2}$ Korea Aerospace Research Institute, Daejeon 34133, Republic of Korea \\ ${ }^{3}$ National Nanofab Center, Deajeon 34141, Republic of Korea \\ ${ }^{4}$ Department of Biomedical Engineering, Kyung Hee University, Yongin 17104, Republic of Korea
}

(Received April 13, 2018; accepted March 20, 2019)

Keywords: capacitive humidity sensor, integrated circuit, capacitance-to-digital converter (CDC), deltasigma CDC, chopper stabilization technique

In this paper, a low-noise 16-bit capacitance-to-digital convertor (CDC) for a capacitive humidity sensor is proposed. The proposed sensor interface circuit is implemented as a firstorder incremental delta-sigma structure and directly converts the capacitance change of the humidity sensor to a 16-bit digital code. A switched capacitor (SC) integrator of the deltasigma modulator is adopted in the chopper stabilization technique for a low-noise characteristic. The chopper stabilization technique can avoid the offset and low-frequency flicker noise of an amplifier. The offset cancellation programmable capacitor array of the input stage is used for eliminating the mismatched capacitance from the parasitic capacitor and process variations. The proposed delta-sigma CDC has an accumulator with a simple 16-bit dual counter that converts the delta-sigma modulator output to a 16-bit digital code for reducing the power consumption of the back-end digital processing. The proposed sensor interface circuit is fabricated using the standard $0.18 \mu \mathrm{m}$ complementary metal-oxide-semiconductor process with an active area of $0.66 \mathrm{~mm}^{2}$. The effective resolution of the worst case is $14.4 \mathrm{bits}$, and the active power consumption of the proposed delta-sigma $\mathrm{CDC}$ is $376 \mu \mathrm{W}$ with a $1.8 \mathrm{~V}$ supply.

\section{Introduction}

Humidity is an important parameter in environmental control systems in many fields such as printers, automobiles, medical devices, and semiconductor manufacturing. Humidity sensors can be made small and highly reliable through micro-electromechanical system (MEMS) technology. Humidity sensors are capacitive and resistive depending on the humidity sensing method used. ${ }^{(1)}$ A resistive humidity sensor can yield a high resolution. However, a resistive humidity sensor requires correction in accordance with the temperature change, and a low relative humidity is difficult to detect. A capacitive humidity sensor does not require temperature compensation, has relatively linear characteristics according to the relative "Corresponding author: e-mail: hhko@cnu.ac.kr https://doi.org/10.18494/SAM.2019.2272 
humidity change from all the relative humidity ranges, and provides a fast response owing to its thin-film structure. With these advantages, capacitive humidity sensors are typically used for relative humidity sensing. ${ }^{(1,2)}$ To use the capacitive humidity sensors in precise environmental control applications, a high-performance sensor interface circuit is required to sense small capacitance variations in the humidity sensor.

Conventional sensor interface circuits convert the capacitance to a voltage signal through a capacitive sensing amplifier (CSA) and subsequently convert the voltage signal from an analogto-digital converter (ADC) to a digital signal. In previous studies, some direct convertible circuit schemes without voltage signal conditioning were proposed. The direct convertible circuit schemes, which are, among others, successive approximation register (SAR) capacitanceto-digital convertors (CDCs), capacitance-to-frequency convertors, and delta-sigma CDCs, can reduce the power and area required for the CSA. ${ }^{(3-11)}$ The SAR CDC consisting of a digitalto-analog convertor (DAC), a comparator, and some digital blocks can yield a low power, a fast conversion, and a small area. However, its resolution is limited owing to the capacitance mismatch and the noise from the comparator. ${ }^{(3)}$ The capacitance-to-frequency (or period) convertor has a simple structure, is suitable for a small area, and consumes a low power. However, the frequency (or period) output is an incomplete digital signal. The frequency (or period) requires an additional conversion to a digital code for back-end digital systems. The additional conversion implies further power consumption.

The delta-sigma CDC is used to obtain a low noise and a high resolution through its noise-shaping characteristic. The higher the order of the modulator, the better the low-noise characteristics; however, the higher the order of modulation, the more power is consumed.

In this paper, a capacitive humidity sensor interface circuit using an incremental delta-sigma CDC scheme is proposed. The proposed delta-sigma CDC adopts a first-order delta-sigma modulator for a low power consumption and a high resolution. The low-noise characteristics of the proposed delta-sigma CDC are obtained using other low-noise techniques instead of higherorder modulators. The dominant noise is primarily caused by a low-frequency flicker $(1 / f)$ noise and the parasitic input capacitance. The proposed first-order delta-sigma modulator reduces the low-frequency $1 / f$ noise using a chopper stabilization technique. Furthermore, the parasitic input capacitance is reduced by the parasitic cancellation capacitance of the input stage. The proposed delta-sigma CDC is designed to adjust the input range to cover the various ranges of capacitive humidity sensors. ${ }^{(12,13)}$ The 1-bit modulated signals of the delta-sigma modulator are finally output by a 16-bit code by the accumulator.

\section{Operation of Proposed Capacitive Humidity Sensor Interface Circuit}

The architecture of the proposed 16-bit delta-sigma CDC is shown below in Fig. 1. The proposed 16-bit delta-sigma CDC consists of a voltage reference, a relaxation oscillator, a timing generator, an offset cancellation input stage, a delta-sigma modulator, and an accumulator.

The reference voltage and timing clock for operating the delta-sigma CDC are generated by the internal blocks. The timing generator has a $4 \mathrm{MHz}$ internal relaxation oscillator and uses the clock by dividing it. The mismatch and parasitic capacitances of the input humidity sensor 


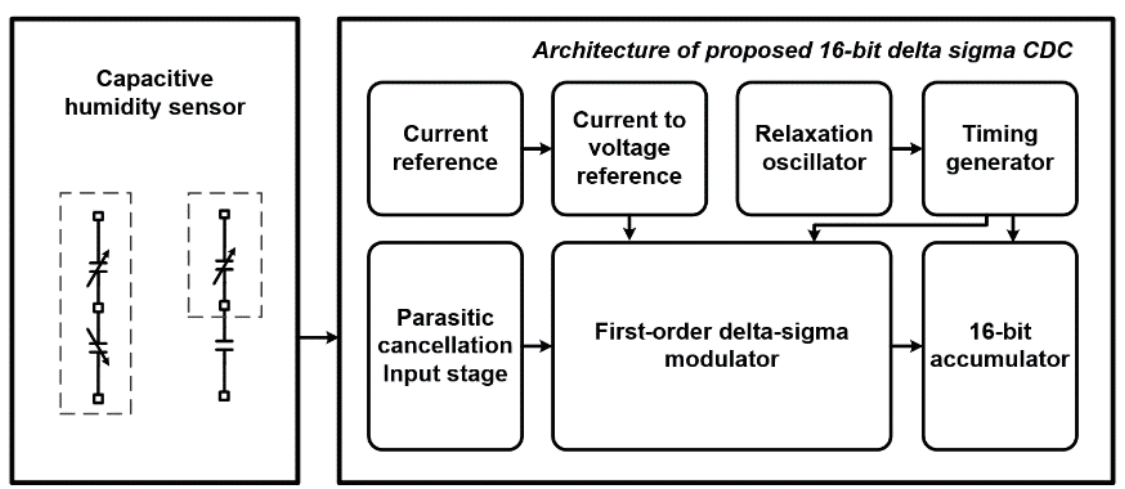

Fig. 1. Architecture of the proposed 16-bit delta-sigma CDC.

are cancelable with the parallel-connected programmable capacitors at the input stage of the proposed delta-sigma CDC. The first-order delta-sigma modulator outputs a 1-bit modulated signal. The accumulator counts the 1-bit modulated signal as a 16-bit code and outputs it.

The schematic of the proposed first-order delta sigma modulator for the capacitive humidity sensor is shown in Fig. 2. The first-order delta-sigma modulator consists of a switched capacitor (SC) integrator, a comparator, and programmable capacitors. The first-order delta-sigma modulator directly converts a capacitance change to digital signals using the SC integrator. The chopper stabilization technique was applied to the integrator of the delta-sigma modulator for low-noise characteristics. The chopper stabilization technique can modulate the $1 / f$ noise of a low-frequency signal band to a high-frequency out band. The chopping clocks of the integrator used the nonoverlapped phases $\mathrm{P} 3$ and $\mathrm{P} 4$.

The first-order delta sigma modulator is operated in two nonoverlapped phases ( $\mathrm{P} 1$ and P2). The capacitive humidity sensor drives the internal or external voltage. The input range is determined by the reference capacitor and driving voltage. In P1, the charges are stored in the sensor and reference capacitors. During P2, the amplifier integrates the charges through a feedback capacitor. The comparator output signal adjusts the driving voltage of CREF to generate the integrator output $V R E F$ in each phase. When the output of the comparator is high, $C R E F$ carries a negative charge to the integrator, while it transfers a positive charge when the output is low. Subsequently, the amount of integrated charge, delivered by the sensor and reference capacitor, becomes increasingly closer to zero. The input range was calculated using the charge equation and the saturation operation of the integrator when switching between P1 and P2. The charge equation when switching from P1 to P2 is shown as

$$
\begin{aligned}
\Delta V= & \frac{(V T O P 1-V T O P 2) \cdot\left(C_{0}+\Delta C\right)}{C F} \\
& +\frac{(V B O T 1-V B O T 2) \cdot\left(C_{0}-\Delta C\right)+2 \cdot C O M P \cdot V R E F \cdot C R E F}{C F} .
\end{aligned}
$$




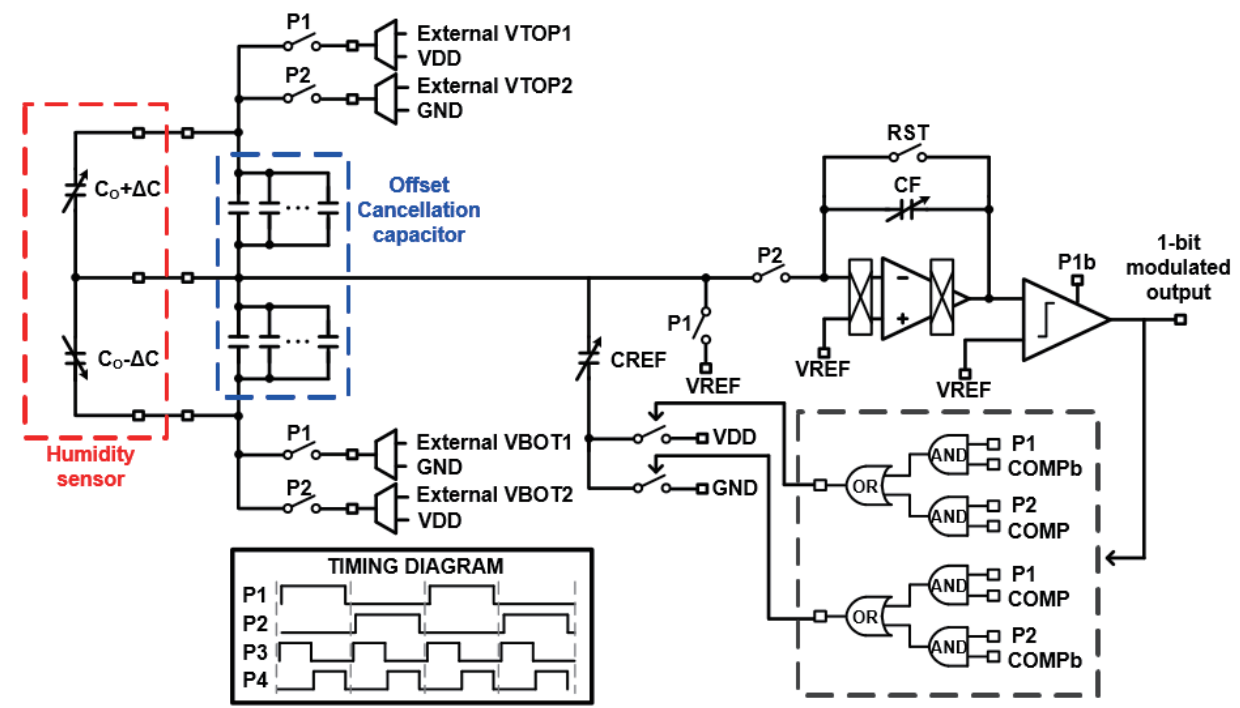

Fig. 2. (Color online) Schematic of the proposed first-order delta-sigma modulator.

The sensor input capacitance when the integrator output saturates indicates the input range. If the comparator output continues to be high, the output of the integrator saturates. The level of the modulated signal is always high even if the charge delivered by CREF is negative. Thus, the maximum capacitance is obtained as

$$
\Delta C<\frac{\{(V T O P 1-V T O P 2)+(V B O T 1-V B O T 2)\} \cdot C_{0}+2 \cdot V R E F \cdot C R E F}{(V T O P 1-V T O P 2)-(V B O T 1-V B O T 2)} .
$$

If the comparator output continues to be low, the output of the integrator saturates. The level of the modulated signal is always low even if the charge delivered by CREF is positive. Thus, the minimum capacitance is obtained as

$$
\Delta C>\frac{\{(V T O P 1-V T O P 2)+(V B O T 1-V B O T 2)\} \cdot C_{0}-2 \cdot V R E F \cdot C R E F}{(V T O P 1-V T O P 2)-(V B O T 1-V B O T 2)} .
$$

When driving voltages are used as internal voltages, the input range is expressed as

$$
-\frac{C R E F}{2}<\Delta C<\frac{C R E F}{2}
$$

$C R E F$ is designed to be a programmable 7-bit capacitor. The programmable $C R E F$ can be applied to humidity sensors of various input ranges. The input range can be increased using external voltages. The internal relaxation oscillator outputs $4 \mathrm{MHz}$ at the main clock. The timing generator divides the main clock to generate the clocks used at the circuit The divided 
clocks are designed with nonoverlapping edges and output to the circuit. Figure 3 shows an accumulator for obtaining a 16-bit digital code. The accumulator of the proposed 16-bit deltasigma CDC has a simple structure consisting of dual 16-bit up-counters and some digital logics. The dual 16-bit up-counter of the proposed accumulator counts the comparator output and clock P1. One of the 16-bit up-counters counts the clock P1 and outputs the end-ofconversion (EOC) signal and a reset signal (RST) at every 16 bits. Another 16-bit up-counter counts the comparator output until the EOC signal increases. When the EOC signal increases, the accumulator outputs a 16-bit digital code. The RST is used in the dual up-counter and integrator of the delta-sigma modulator. The output digital code resolution can be determined to be 12 or 16 bits by selecting the period of the RST. When a fast response and a low resolution are required, the 12-bit resolution should be used.

\section{Measurement Results of Proposed IC}

A fabricated die photograph of the proposed 16-bit delta-sigma CDC is shown in Fig. 4(a). The 16-bit delta-sigma CDC was designed using the standard $0.18 \mu \mathrm{m}$ complementary metaloxide-semiconductor (CMOS) process with an active area of $0.66 \mathrm{~mm}^{2}$ excluding the $\mathrm{I} / \mathrm{O}$ pads. The total power consumption for the 16-bit delta-sigma $\mathrm{CDC}$ is $376 \mu \mathrm{A}$ with a $1.8 \mathrm{~V}$ supply. The digitally controlled 10-bit capacitor array, which is used in our experiment, is also integrated in the die.

Figure 5 shows the measurement environment and the equipment for measuring the proposed circuit. The fabricated chip was implemented on a printed circuit board for the measurement of the circuit. To evaluate the performance of the proposed circuit, the 10-bit digital controlled capacitor array in the chip was used. The humidity sensor used the CL-MC-02 sensor that is a polymer relative humidity capacitor. The digital output is acquired through an Arduino and a PC.

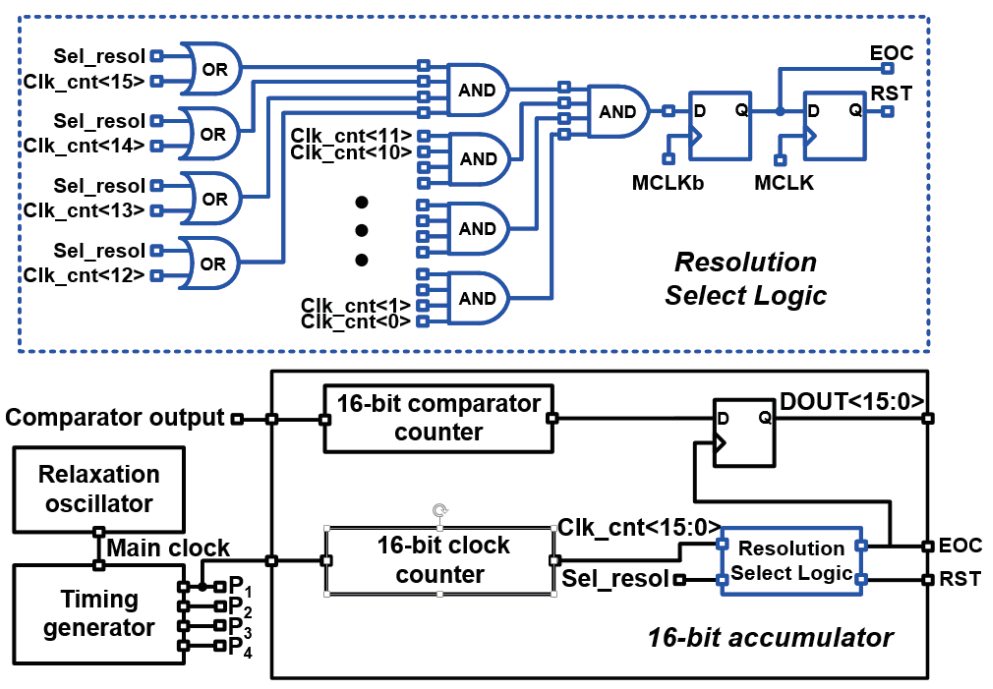

Fig. 3. (Color online) Accumulator for obtaining 16-bit digital code output. 


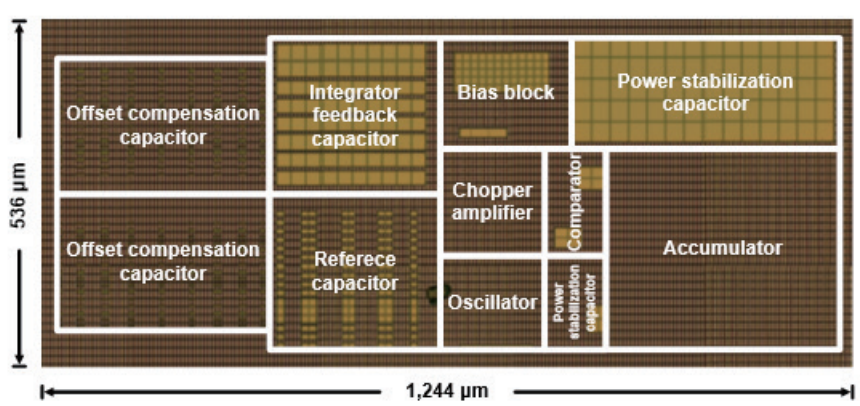

(a)

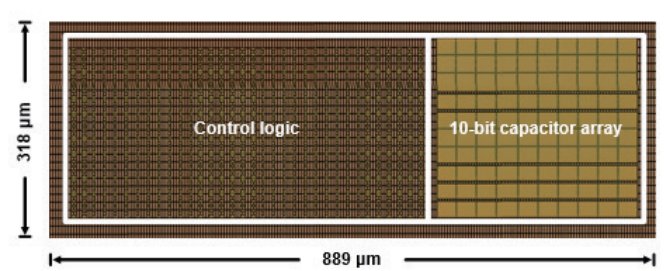

(b)

Fig. 4. (Color online) (a) Die photograph of the 16-bit delta-sigma CDC. (b) Die photograph of the digital controlled 10-bit capacitor array.

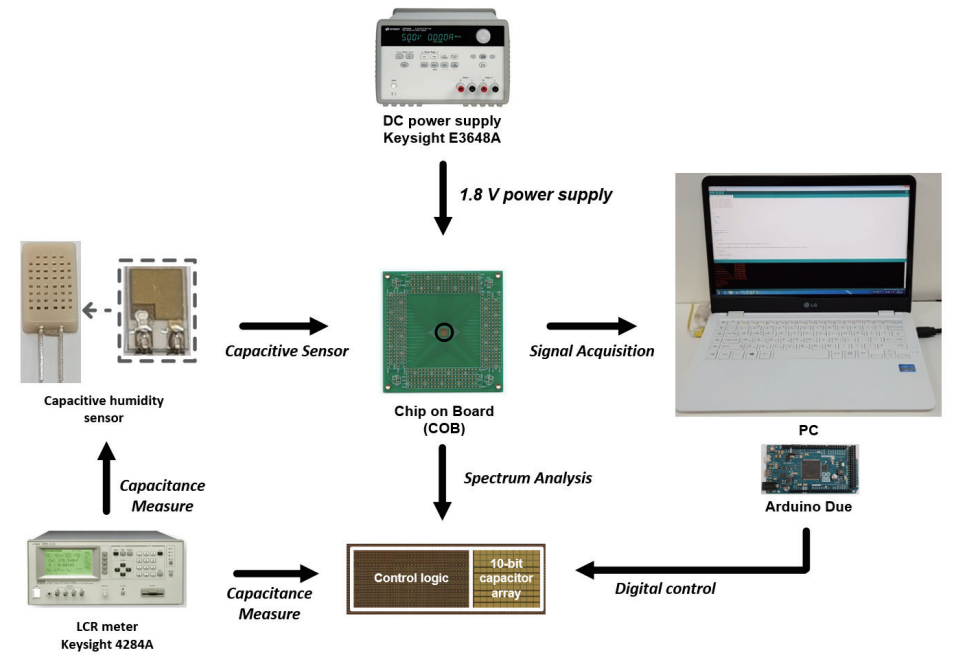

Fig. 5. (Color online) Measurement environment for the 16-bit delta-sigma CDC.

Figure 6 shows the measurement result for the input capacitance changes. To measure linearity, the input capacitor used is a chip capacitor, and the digitally controlled 10-bit capacitor array is used to control the capacitance range from 8.5 to $24.5 \mathrm{pF}$. The external chip capacitor is used as an offset capacitor for the input range setting. In the input range of $10 \mathrm{pF}$, the nonlinearity is calculated to be equal to $1.83 \%$ FSO. Figure 7 shows the standard deviations for 500 samples of each fixed input capacitance. The spot points are the results of using the chopper stabilization technique and the square points turn off the chopper stabilization. The effective resolution is calculated as

$$
\text { Effective resolution }=\log _{2}\left(\frac{\text { Fullscale capacitance input range }}{R M S \text { noise }}\right) \text {. }
$$

When the chopper stabilization technique is used, the effective resolution in the best case is 14.4 bits in the $10 \mathrm{pF}$ input capacitance range. When the chopper stabilization technique is not 


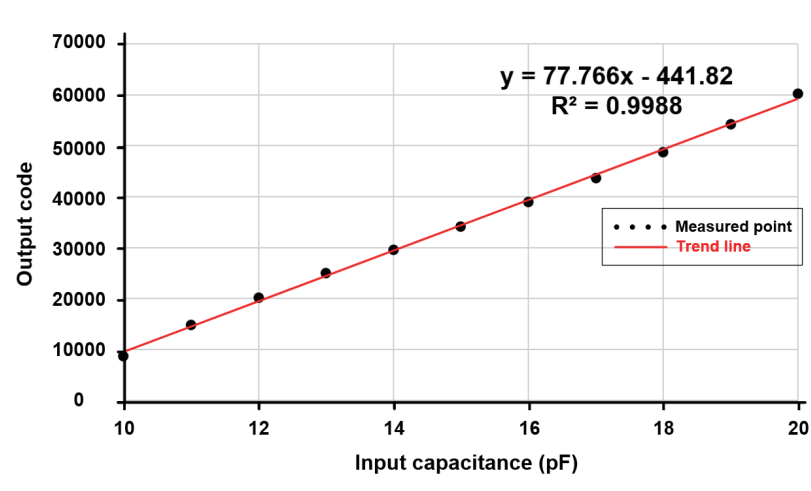

Fig. 6. (Color online) Linearity for the input capacitance changes.

Table 1

Technical specifications of the humidity sensor.

\begin{tabular}{lc}
\hline Model & MC02 \\
\hline Power supply & $10 \mathrm{~V} \mathrm{AC} \mathrm{(max)}$ \\
\hline Operating range & Humidity $0-100 \% \mathrm{RH}$ \\
\hline Nominal capacitance & $\begin{array}{c}120 \pm 10 \mathrm{pF} \\
(25 \mathrm{Celsius}, 60 \% \mathrm{RH})\end{array}$ \\
\hline Average sensitivity & $0.05 \mathrm{pF} / \% \mathrm{RH}$ \\
\hline Humidity effects & $+1 \mathrm{Celsius},+0.025 \mathrm{pF}$ \\
\hline $\begin{array}{l}\text { Return difference of } \\
\text { humidity hysteresis }\end{array}$ & $<1 \% \mathrm{RH}$ \\
\hline Linear error & $< \pm 2 \% \mathrm{RH}$ \\
\hline Operating frequency & $5-100 \mathrm{kHz}$ \\
\hline
\end{tabular}

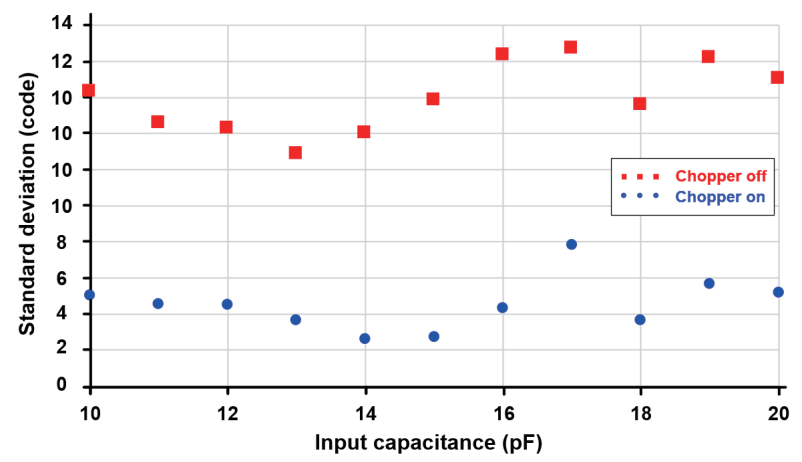

Fig. 7 (Color online) Standard deviation of each fixed input capacitance.

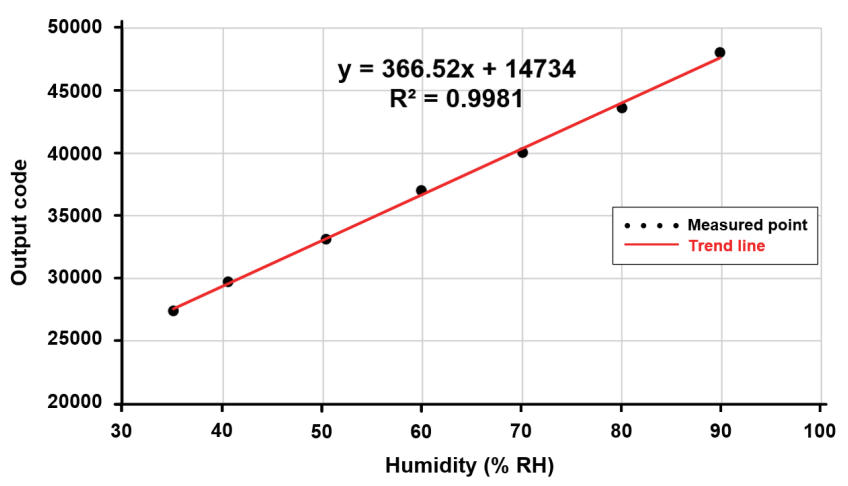

Fig. 8. (Color online) Measurement results for the relative humidity changes.

used, the effective resolution in the best case is 12.16 bits in the $10 \mathrm{pF}$ input capacitance range. When using chopper stabilization techniques, the best resolution in the best case is 2.24 bits more than if it were not.

The proposed delta-sigma CDC with a humidity sensor was measured using a humidity chamber. The technical specification of the humidity sensor is shown in Table 1. Owing to the limited available humidity range of the humidity chamber, the relatively humidity was measured from 35 to $90 \% \mathrm{RH}$ at $25{ }^{\circ} \mathrm{C}$. The measured code outputs increasing with the relatively humidity are shown in Fig. 8.

When the humidity changes from 35 to $90 \% \mathrm{RH}$, the output code is changed to the 20627 code. The 20627 code change means a change of $3.8 \mathrm{pF}$ in capacitance. The sensitivity of the 16-bit delta-sigma CDC with a humidity sensor was measured to be $58 \mathrm{fF} / \% \mathrm{RH}$. The conversion time of the delta-sigma CDC using the 16-bit accumulator is $120 \mathrm{~ms}$. The number of code samples obtained is 500 at each RH point. Figure 9(a) shows the code variations for each RH change in $60 \mathrm{~s}$. The standard deviation for each RH change is shown Fig. 9(b). In the results of the experiment with a humidity sensor, larger noise was measured than in the experiment with the programmable capacitor. Because the humidity chamber maintains the humidity with the circulation of wind, much noise was measured together. 


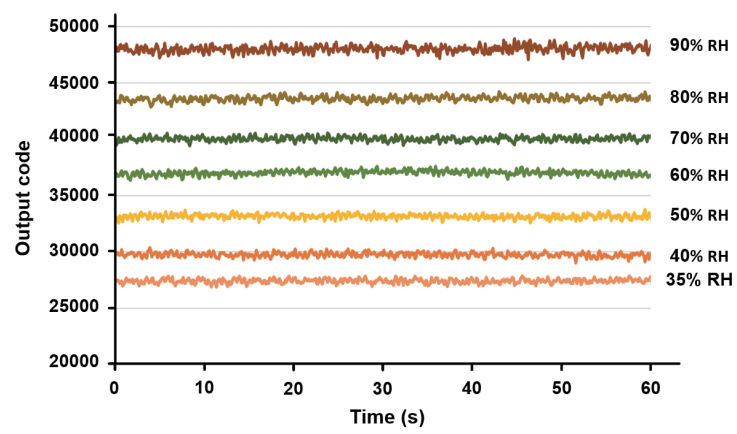

(a)

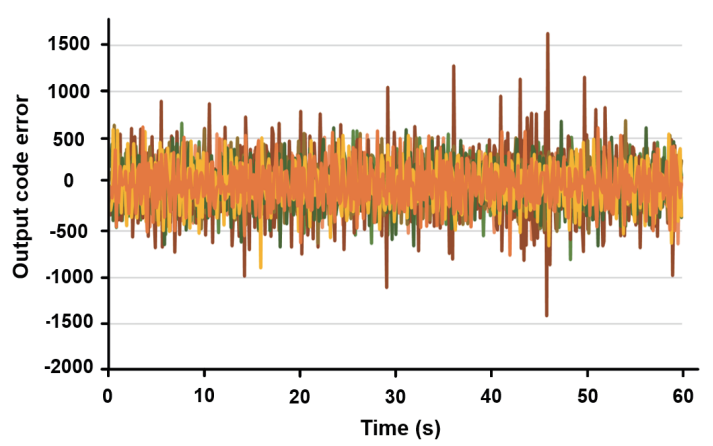

(b)

Fig. 9. (Color online) (a) Output code variations and (b) output code error for each relative humidity change.

Table 2

Performance comparison and summary of parameters.

\begin{tabular}{lcccccc}
\hline & This work & Ref. 3 & Ref. 8 & Ref. 9 & Ref. 10 & Ref. 11 \\
\hline Architecture & 1 st $\Delta \Sigma$ CDC & SAR CDC & 2nd $\Delta \Sigma$ CDC & 3rd $\Delta \Sigma$ CDC & 2nd $\Delta \Sigma$ CDC & 3rd $\Delta \Sigma$ CDC \\
Technology $(\mu \mathrm{m})$ & 0.18 & 0.35 & 0.16 & 0.16 & 0.18 & 0.35 \\
Supply $(\mathrm{V})$ & 1.8 & 3.3 & 1.8 & $1.2-1.8$ & 2.6 & 3.3 \\
Power $(\mu \mathrm{W})$ & 376 & 303 & 10.53 & $10.32($ at $1.2 \mathrm{~V})$ & 2340 & 15000 \\
Area $\left(\mathrm{mm}^{2}\right)$ & 0.66 & 0.07 & & & 0.67 & 5.58 \\
Effective resolution $(\mathrm{bit})$ & 14.4 & 12.5 & 13.3 & 12.85 & 17.4 & 17.2 \\
Capacitance range $(\mathrm{pF})$ & 20 (programmable) & 16 & $0.8 \pm 0.4$ & $0.8 \pm 0.26$ & 10 & 10 \\
\hline
\end{tabular}

A performance summary of the parameters and comparisons is shown in Table 2. The effective resolution from Refs. 8 and 9 was calculated and is shown in Table 2 for comparison. The effective resolution of the 16-bit delta-sigma CDC is 14.4 bits with a $10 \mathrm{pF}$ input capacitance range. In Table 2, the proposed 16-bit delta-sigma CDC uses a simple first-order modulator architecture to obtain a low power and small-area characteristics, and has a high effective resolution of 14.4 bits by obtaining low noise characteristics. Also, the widest programmable input capacitance range among previous studies can be used in the humidity sensor applications of various input ranges.

\section{Conclusions}

A low-noise 16-bit delta-sigma CDC for sensing a capacitive humidity sensor was presented. The proposed 16-bit CDC used the delta-sigma modulator that adopted a chopper stabilization technique. The proposed delta-sigma CDC yielded a small area and a low power consumption by using a simple first-order delta-sigma modulator. As the chopper stabilization technique improved the effective resolution by 2.24 bits, the proposed delta-sigma CDC had a high effective resolution of 14.4 bits. To reduce the back-end digital processing power, the proposed delta-sigma CDC was fully integrated with the accumulator. For use with various capacitive humidity sensors of different input ranges, the proposed delta-sigma CDC was designed to 
demonstrate a programmable wide input capacitance range. The chip was fabricated using the $0.18 \mu \mathrm{m}$ CMOS process with an active area of $0.66 \mathrm{~mm}^{2}$. The total power consumption of the proposed CDC was $376 \mu \mathrm{W}$ with a $1.8 \mathrm{~V}$ supply.

\section{Acknowledgments}

This research was supported by the NanoMaterial Technology Development Program through the National Research Foundation of Korea (NRF) funded by the Ministry of Science, ICT, and Future Planning (2015M3A7B7045527). This research was also supported by a grant from Kyung Hee University in 2017 (KHU-20171202).

\section{References}

1 H. Farahani, R. Wagiran, and M. N. Hamidon: MDPI Sens. J. 14 (2014) 7881.

2 R. Fenner and E. Zdankiewicz: IEEE Sens. J. 1 (2001) 309.

3 H. Omran, M. Arsalan, and K. N. Salama: Sens. Actuators, A 216 (2014) 43.

4 Z. Tan, S. H. Shalmany, G. C. Meijer, and M. A. Pertijs: IEEE J. Solid-State Circuits 47 (2012) 1703.

5 P. Bruschi, N. Nizza, and M. Piotto: IEEE J. Solid-State Circuits 42 (2007) 1884.

6 V. Matko and M. Milanović: Sens. Actuators, A 220 (2014) 262.

7 D. Y. Shin, H. Lee, and S. Kim: IEEE Trans. Circuits. Syst. II, Exp. Briefs 58 (2011) 90.

8 Z. Tan, R. Daamen, A. Humbert, K. Souri, Y. Chae, Y. V. Ponomarev, and M. A. Pertijs: IEEE ASSCC (IEEE, 2011) $105-108$

9 Z. Tan, Y. Chae, R. Daamen, A. Humbert, Y. V. Ponomarev, and M. A. Pertijs: 2012 Symp. VLSI Circuits (IEEE, 2012) 24-25.

10 Y. Jung, Q. Duan, and J. Roh: IEEE Trans. Circuits. Syst. II, Exp. Briefs 64 (2017) 1122.

11 S. Xia, K. Makinwa, and S. Nihtianov: 2012 IEEE Int. Solid-State Circuits Conf. (IEEE, 2012) 198-200.

12 L. Gu and Q. A Huang: Sens. Actuators, B 99 (2004) 491.

13 S. Chatzandroulis, A. Tserepi, D. Goustouridis, P. Normand, and D. Tsoukalas: Microelectron. Eng. 61-62 (2002) 955.

\section{About the Authors}

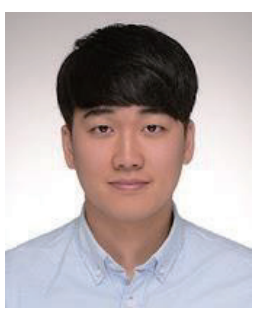

Byeoncheol Lee received his B.S. degree in Electronics Engineering from Chungnam National University, Daejeon, Republic of Korea, in 2017, where he is currently pursuing his M.S. degree. His current research interests are in the design of CMOS analog and mixed-mode integrated circuits.

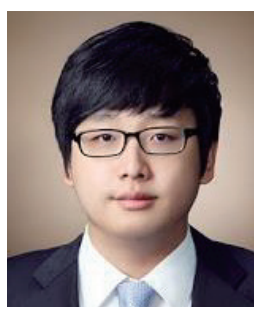

Hyungseup Kim received his B.S. degree in Electronics Engineering from Chungnam National University, Daejeon, Republic of Korea, in 2014, where he is currently pursuing his Ph.D. degree. His current research interests are in the design of sensor interface circuits, biosignal acquisition circuits, secure integrated circuits, data converters, and mixed-mode integrated circuits. 


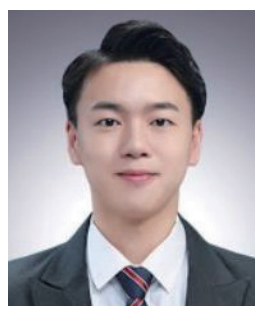

Jaesung Kim received his B.S. degree in Electronics Engineering from Chungnam National University, Daejeon, Republic of Korea, in 2018, where he is currently pursuing his M.S. degree. His current research interests are in the design of CMOS analog and mixed-mode integrated circuits.

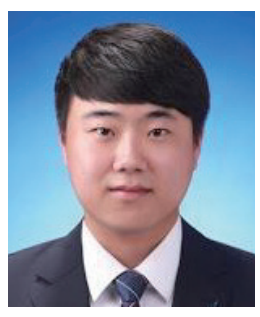

Kwonsang Han received his B.S. degree in Electronics Engineering from Chungnam National University, Daejeon, Republic of Korea, in 2018, where he is currently pursuing his M.S. degree. His current research interests are in the design of CMOS analog and mixed-mode integrated circuits.

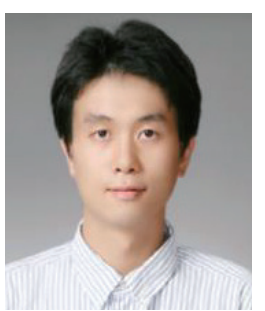

Sangyoun Shin received his B.S. and M.S. degrees from Sogang University, Republic of Korea, in 1999 and 2001, respectively. From 2001 to 2008, he worked with Samsung Electronics as a senior engineer. In 2008, he joined the Satellite Payload Development Division at the Korea Aerospace Research Institute, where he is currently a payload electronics senior researcher. His current research interests are in the design of high-resolution lunar camera electronics of the Korea Pathfinder Lunar Orbiter.

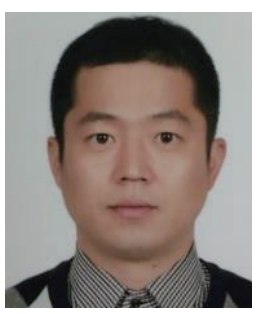

Gyungtae Kim received his B.S. degree from the Department of Electrical Engineering at Inha University, Korea, in 2004, and his Ph.D. degree from the Department of Electronics Engineering at Chungnam National University, Korea, in 2016. From 2004 to 2011, he was with SK Hynix as a senior engineer. Since 2011, he has worked for the National Nanofab Center in Korea as a senior researcher. His research interests include MEMS sensors and analog integrated circuit design.

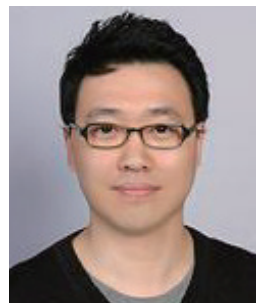

Woo Suk Sul received his B.S., M.S., and Ph.D. degrees from the Department of Electronic Engineering at Dongguk University, Korea, in 2000, 2002, and 2011, respectively. From 2004 to 2011, he developed mixed-signal and RF devices at the Hynix/Magnachip Semiconductor System IC R\&D Center. Since 2007, he has worked at the National NanoFab Center in Korea as a senior researcher. His research interest is in developing semiconductor detectors for radiation detection and medical imaging. 


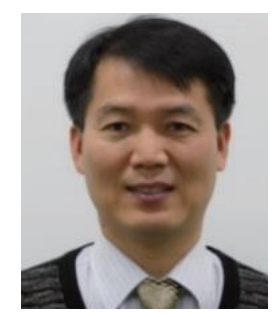

Boung Ju Lee received his B.S., M.S., and Ph.D. degrees from the Department of Metallurgical Engineering at Seoul National University, Seoul, Korea, in 1992, 1994, and 1997, respectively. From 1997 to 2008, he was a principal technology development engineer of Hynix Semiconductor in Korea, Chartered Semiconductor in Singapore, and Samsung Electronics in Korea, successively. Since 2008, he has worked in the National NanoFab Center in Korea as a principal researcher. His research interest is in the process integration and development of next-generation semiconductor devices and sensors.

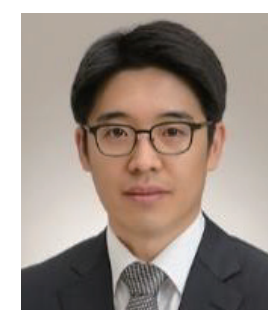

Sangmin Lee received his B.S. and Ph.D. degrees from the School of Electrical Engineering at Seoul National University, Republic of Korea, in 2005 and 2013, respectively. From 2013 to 2016, he worked as a research staff member at Samsung Advanced Institute of Technology (SAIT), Samsung Electronics, Inc. in the Republic of Korea. In 2016, he joined the Department of Biomedical Engineering, Kyung Hee University, Yongin, Republic of Korea, where he is currently an assistant professor. His main research interest includes biomedical sensors and systems for applications in Internet of Things (IoT) devices.

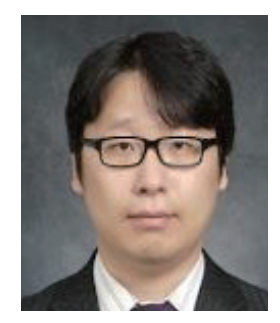

Hyoungho Ko received his B.S. and Ph.D. degrees in Electrical Engineering from Seoul National University, Republic of Korea, in 2003 and 2008, respectively. From 2008 to 2010, he worked with Samsung Electronics as a senior engineer. In 2010, he joined the Department of Electronics Engineering at Chungnam National University, Republic of Korea, where he is currently an associate professor. His current research interests are in the design of CMOS analog and mixed-mode integrated circuits. 